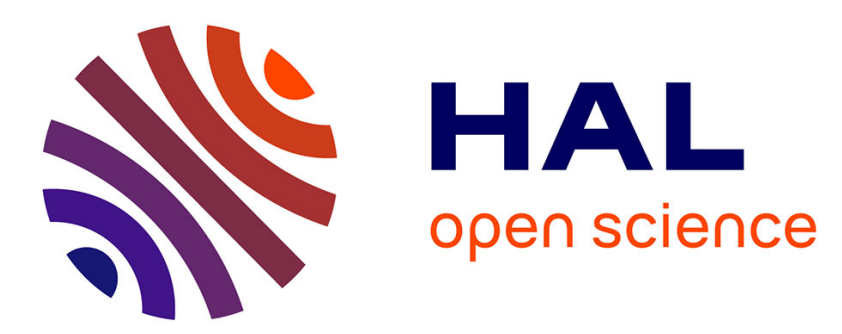

\title{
A mathematical analysis of the resonance of the finite thin slots
}

\author{
Marianne Clausel, Marc Duruflé, Patrick Joly, Sébastien Tordeux
}

\section{To cite this version:}

Marianne Clausel, Marc Duruflé, Patrick Joly, Sébastien Tordeux. A mathematical analysis of the resonance of the finite thin slots. Applied Numerical Mathematics, 2006, 56 (10-11), pp.1432-1449. hal-00404068

\section{HAL Id: hal-00404068 \\ https://hal.science/hal-00404068}

Submitted on 15 Jul 2009

HAL is a multi-disciplinary open access archive for the deposit and dissemination of scientific research documents, whether they are published or not. The documents may come from teaching and research institutions in France or abroad, or from public or private research centers.
L'archive ouverte pluridisciplinaire $\mathbf{H A L}$, est destinée au dépôt et à la diffusion de documents scientifiques de niveau recherche, publiés ou non, émanant des établissements d'enseignement et de recherche français ou étrangers, des laboratoires publics ou privés. 


\title{
A mathematical analysis of the resonance of the finite thin slots
}

\author{
M. Clausel ${ }^{a, b}$, M. Duruflé ${ }^{a, b}$, P. Joly ${ }^{a, b}$, S. Tordeux ${ }^{a, b, c, *}$ \\ ${ }^{a}$ Projets POems: unité de recherche mixte 2706 CNRS-ENSTA-INRIA \\ ${ }^{\mathrm{b}}$ INRIA, Projet Ondes, Batiment 13, INRIA, Domaine de Voluceau - \\ Rocquencourt - B.P. 105, 78153 Le Chesnay Cedex, France \\ ${ }^{\mathrm{c}}$ ENSTA, 32 Boulevard Victor 75739 Paris cedex 15, France
}

\begin{abstract}
In this article we are interested in the asymptotic behaviour of the solution of a wave propagation problem in a domain including a thin slot. Some rate of convergences are obtained and illustrated by numerical results.
\end{abstract}

Key words: thin slot, slit, resonance, Helmholtz, wave equation, 1991 MSC: 35J05, 65N15, 78M30, 78M35

\section{Introduction and position of the problem}

The analysis of the propagation of waves in media including thin slots poses, as for any PDE problem involving a small scale (here the width of the slot), some difficulties from the numerical point of view and raises exciting related mathematical questions. Moreover, this kind of problem appears in a lot of applications in acoustics and electromagnetism and has already generated an abundant literature in the engineering community.

In [1], we studied the case of the 2D time harmonic wave equation with Neumann boundary conditions (which is physically relevant in acoustics and electromagnetism) and investigated the question of building an approximate model, in view of its numerical discretization, consisting of coupling a 1D

\footnotetext{
* Corresponding author. Adress: Projet Ondes, Batiment 13, INRIA, Domaine de Voluceau - Rocquencourt - B.P. 10578153 Le Chesnay Cedex France. Email: sebastien.tordeux@inria.fr
} 
model for the slot to a $2 \mathrm{D}$ model for the rest of the domain of propagation. We proposed a coupling strategy that we analyzed in detail in the case of a semi-infinite straight slot. The basic ingredient of the analysis is of course the study of the asymptotic behaviour of the solution when the width $\varepsilon$ of the slot tends to 0 . The analysis of the same question for a finite slot, that we wish to investigate in this paper, appears to be more delicate. One has in particular to face the question of resonant frequencies (or equivalently critical slot lengthes), a phenomenon which is already known by engineers [2] and that we propose to analyze from both analytical and numerical points of view.

Let us first present our model problem and begin by the description of the

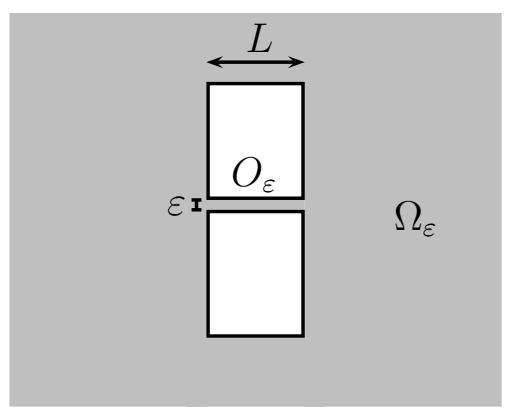

Fig. 1. Geometry of the propagation domain with the slot

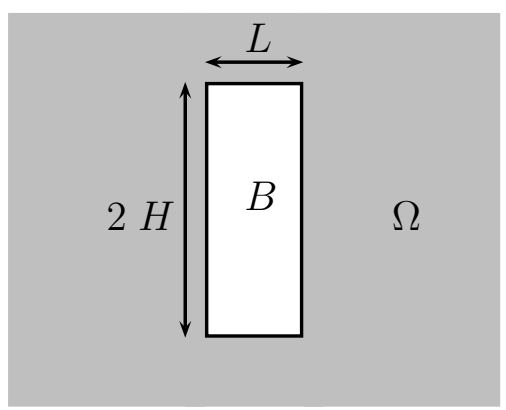

Fig. 2. Geometry of the propagation domain without the slot

geometry of the problem. We assume that we are in $2 \mathrm{D}(\mathbf{x}=(x, y)$ will denote the current point of $\mathbb{R}^{2}$ ) and that the domain of propagation $\Omega_{\varepsilon}$ is given by (with $L>0$ and $0<\varepsilon<H$ ):

$$
\begin{aligned}
& \overline{\Omega_{\varepsilon}}=\bar{\Omega} \cup \overline{O_{\varepsilon}}, \quad \Omega=\mathbb{R}^{2} \backslash B \\
& B=[0, L] \times[-H, H], \quad \text { (a rectangle) } \\
& \left.O_{\varepsilon}=\right] 0, L[\times] 0, \varepsilon[, \quad \text { (a straight finite slot) }
\end{aligned}
$$

We shall call "end points" of the slot the two points $\mathbf{A}^{-}=\left(a^{-}, b^{-}\right) \equiv(0,0)$ and $\mathbf{A}^{+}=\left(a^{+}, b^{+}\right) \equiv(L, 0)$ and denote $\mathbf{A}$ the middle of $\left[\mathbf{A}^{-}, \mathbf{A}^{+}\right]$.

We assume that a time harmonic wave of pulsation $\omega>0(c=1)$ is emitted by a source term $f$ with compact support $F$ included inside the open set $\Omega$ :

$$
\begin{cases}\text { Find } u^{\varepsilon} \in H^{1}\left(\Omega_{\varepsilon}\right) \text { outgoing such that: } & \\ -\Delta u^{\varepsilon}-\omega^{2} u^{\varepsilon}=f, & \text { in } \Omega_{\varepsilon} \\ \frac{\partial u^{\varepsilon}}{\partial n}=0, & \text { on } \partial \Omega_{\varepsilon} .\end{cases}
$$

It is classical and useful to reduce the problem to a bounded domain thanks to a transparent boundary condition on the circle $\Gamma_{R}=\{\mathbf{x} \in \Omega /|\mathbf{x}-\mathbf{A}|=R\}$, 
where the radius $R>0$ is large enough so that the obstacle and the support $F$ of $f$ are contained in $\Omega_{R}:=\{\mathbf{x} \in \Omega /|\mathbf{x}-\mathbf{A}|<R\}$. A Dirichlet to Neumann transparent boundary condition can be put on $\Gamma_{R}$ as:

$$
\frac{\partial u^{\varepsilon}}{\partial n}+T_{R} u^{\varepsilon}=0, \quad \text { on } \Gamma_{R}
$$

where the operator $T_{R}$ from $H^{\frac{1}{2}}\left(\Gamma_{R}\right)$ to $H^{-\frac{1}{2}}\left(\Gamma_{R}\right)$ is given by:

$$
\left\{\begin{array}{l}
T_{R} u=\sum_{n=-\infty}^{+\infty} \mu_{n}^{R}(\omega) u_{n}^{R} \psi_{n}^{R}, \\
u_{n}^{R}=\int_{0}^{2 \pi} u(R, \theta) \psi_{n}^{R}(\theta) R d \theta, \quad \psi_{n}^{R}(\theta)=\sqrt{1 /(2 \pi R)} \exp \operatorname{in} \theta, \\
\mu_{n}^{R}(\omega)=-\omega \frac{\left(H_{|n|}^{(1)}\right)^{\prime}(\omega R)}{H_{|n|}^{(1)}(\omega R)}, \quad \operatorname{Re}\left(\mu_{n}^{R}(\omega)\right) \geqslant 0 \text { and } \operatorname{Im}\left(\mu_{n}^{R}(\omega)\right) \leqslant 0 .
\end{array}\right.
$$

In this paper, we are interested in the behaviour of the solution $u^{\varepsilon}$ when the width $\varepsilon$ tends to 0 . Intuition suggests that $u^{\varepsilon}$ should converge to the solution $u^{0}$ of the "slotless" problem:

$$
\begin{cases}\text { Find } u^{0} \in H^{1}(\Omega) \text { outgoing such that: } & \\ -\Delta u^{0}-\omega^{2} u^{0}=f, & \text { in } \Omega \\ \frac{\partial u^{0}}{\partial n}=0, & \text { on } \partial \Omega .\end{cases}
$$

This is what we proved in [1] in the case of the semi-infinite slot (i. e. $L=+\infty$ ), where we proved moreover that

$$
u^{\varepsilon}-u^{0}=O(\varepsilon), \quad \text { in } L_{l o c}^{2} .
$$

In the case of the finite slot, we shall see that such an estimate is correct except for a set of exceptional frequencies $\omega$, called resonant frequencies, corresponding to:

$$
\omega L=\ell \pi, \quad \ell \in \mathbb{N}^{*},
$$

for which the convergence is much slower. More precisely:

$$
u^{\varepsilon}-u^{0}=O\left(\frac{1}{|\log \varepsilon|}\right), \quad \text { in } L_{l o c}^{2}
$$


Inside the slot, the solution remains bounded for non resonant frequencies but blows up for resonant frequencies.

The outline of the rest of the paper is as follows. In section 2, we investigate the case of non resonant frequencies and prove, in this case, the convergence of $u^{\varepsilon}$ to $u^{0}$. In section 3 , we investigate the case of resonant frequencies through numerical results. Our results suggest that the solution $u^{\varepsilon}$ slowly converges outside the slot to the limit $u^{0}$-see equation (10)- and blows up inside the slot. Finally, in section 4 , we give a formal analysis of these observations, using the technique of matched asymptotic expansions.

\section{The case of non resonant frequencies: mathematical analysis}

The aim of this section is to prove the following theorem, which formulates in a precise way one of the assertions in the introduction. Note that estimates (11) can be shown to be generically optimal as in [1]. We conjecture that the constant $C$ blows up as $1 / d(\omega)$ where $d(\omega)$ denotes the distance to the set of resonant frequencies.

Theorem 1 For all $\omega$, L, such that (9) does not hold and for all $R$ in $\mathbb{R}^{*,+}$ and for any $F$ closed subset of $\Omega$ there exists $C \in \mathbb{R}$ and $\varepsilon_{0}>0$ such that for all $0<\varepsilon<\varepsilon_{0}$ and $f \in L^{2}(\Omega)$ with support included in $F$, we have:

$$
\left\{\begin{array}{l}
\left\|u^{\varepsilon}-u^{0}\right\|_{H^{1}\left(\Omega_{R}\right)} \leqslant C(\omega, L, R, F) \varepsilon \sqrt{|\log \varepsilon|}\|f\|_{L^{2}(\Omega)}, \\
\left\|u^{\varepsilon}-u^{0}\right\|_{L^{2}\left(\Omega_{R}\right)} \leqslant C(\omega, L, R, F) \varepsilon\|f\|_{L^{2}(\Omega)} .
\end{array}\right.
$$

and

$$
\left\|u^{\varepsilon}\right\|_{L^{2}\left(O_{\varepsilon}\right)} \leqslant C(\omega, L, F) \sqrt{\varepsilon}\|f\|_{L_{2}(\Omega)}
$$

2.1 Reformulation of the problem (4) in the domain $\Omega_{R}$.

Preliminary material. We first recall precise trace theorems on:

$$
\left.\Sigma_{\varepsilon}=\Sigma_{\varepsilon}^{-} \cup \Sigma_{\varepsilon}^{+}, \quad \Sigma_{\varepsilon}^{-}=\{0\} \times\right] 0 ; \varepsilon\left[, \quad \Sigma_{\varepsilon}^{+}=\{0\} \times\right] 0 ; \varepsilon[.
$$

In what follows, we shall often identify $\Sigma_{\varepsilon}^{-}$and $\Sigma_{\varepsilon}^{+}$to the segment $] 0 ; \varepsilon[$.

We introduce $\left(\left(w_{n}^{\varepsilon}\right)\right)_{n \in \mathbb{N}}$, the orthogonal basis of $H^{s}(] 0 ; \varepsilon[)$ for $s \in\left[0 ; \frac{3}{2}[\right.$ :

$$
w_{0}^{\varepsilon}(y)=\sqrt{1 / \varepsilon} \quad \text { and } \quad w_{n}^{\varepsilon}(y)=\sqrt{2 / \varepsilon} \cos \frac{n \pi y}{\varepsilon}, \quad \forall n \in \mathbb{N}^{*} .
$$


For all $\mathbf{u}$ in $L^{2}\left(\boldsymbol{\Sigma}_{\varepsilon}\right)$, we denote by $\mathbf{u}_{n}^{\varepsilon} \in \mathbb{C}^{2}$ the vector of the coefficients (in this basis) of the respective restrictions of $\mathbf{u}$ to $\Sigma_{\varepsilon}^{-}$and $\Sigma_{\varepsilon}^{+}$:

$$
\mathbf{u}_{n}^{\varepsilon}=\left(\int_{\Sigma_{\varepsilon}^{-}} \mathbf{u} w_{n}^{\varepsilon} d \sigma, \int_{\Sigma_{\varepsilon}^{+}} \mathbf{u} w_{n}^{\varepsilon} d \sigma\right)^{t}
$$

that allows us to characterize the spaces $H^{s}\left(\boldsymbol{\Sigma}_{\varepsilon}\right)$ for $0 \leqslant s<\frac{3}{2}$ :

$$
\mathbf{u} \in H^{s}\left(\boldsymbol{\Sigma}_{\varepsilon}\right) \Longleftrightarrow\|\mathbf{u}\|_{H^{s}\left(\boldsymbol{\Sigma}_{\varepsilon}\right)}^{2}=\sum_{n=0}^{+\infty}\left(1+\pi^{2} n^{2} / \varepsilon^{2}\right)^{s}\left|\mathbf{u}_{n}^{\varepsilon}\right|^{2}<+\infty
$$

The dual space $\left(H^{s}\left(\boldsymbol{\Sigma}_{\varepsilon}\right)\right)^{*}$ can be defined analogously if one replaces the integrals by duality brackets in (15). We shall also use the following semi-norm in $H^{s}\left(\boldsymbol{\Sigma}_{\varepsilon}\right)$ :

$$
\|\mathbf{u}\|_{H_{*}^{s}\left(\boldsymbol{\Sigma}_{\varepsilon}\right)}^{2}=\sum_{n=1}^{+\infty}\left(1+\pi^{2} n^{2} / \varepsilon^{2}\right)^{s}\left|\mathbf{u}_{n}^{\varepsilon}\right|^{2}
$$

From now on, we shall often denote by $\mathbf{u}$ the trace on $\boldsymbol{\Sigma}_{\varepsilon}$ of a function $u \in$ $H_{l o c}^{1}(\Omega)$ and by $\mathbf{u}_{n}^{\varepsilon}$ the vectors of its generalized Fourier coefficients (see (15)). One can easily adapt the proof of [1] to obtain the following results.

Lemma 2 For any neighborhood $V$ of $A^{-}$and $A^{+}$, for $s \in \mathbb{R}^{+}$, there exists $C(s) \in \mathbb{R}$ and $\varepsilon_{0}>0$ such that for all $u \in H^{1+s}(\Omega \cap V)$ and $\left.\left.\varepsilon \in\right] 0 ; \varepsilon_{0}\right]$ :

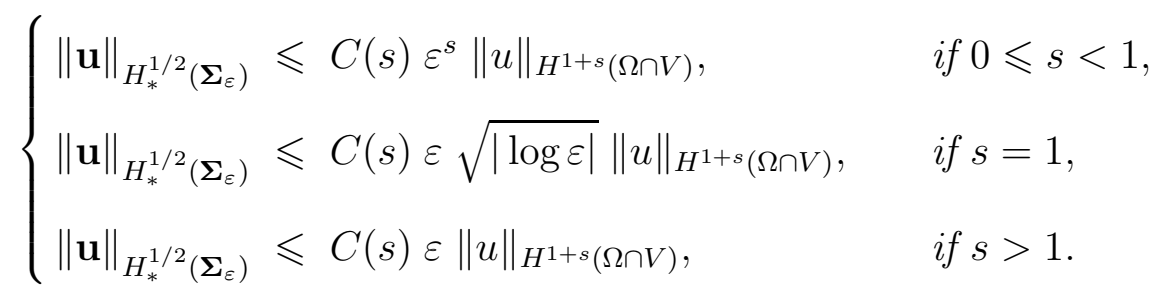

Moreover, for all $u \in H^{\frac{1}{2}+s}(\Omega \cap V)$ and $\left.\left.\varepsilon \in\right] 0 ; \varepsilon_{0}\right]$ :

$$
\begin{cases}\left|\mathbf{u}_{0}^{\varepsilon}\right| \leqslant C(s) \varepsilon^{s}\|u\|_{H^{\frac{1}{2}+s}(\Omega \cap V)}, & \text { if } 0<s<\frac{1}{2}, \\ \left|\mathbf{u}_{0}^{\varepsilon}\right| \leqslant C(s) \sqrt{\varepsilon|\log \varepsilon|}\|u\|_{H^{\frac{1}{2}+s}(\Omega \cap V)}, & \text { if } s=\frac{1}{2}, \\ \left|\mathbf{u}_{0}^{\varepsilon}\right| \leqslant C(s) \sqrt{\varepsilon}\|u\|_{H^{\frac{1}{2}+s}(\Omega \cap V)}, & \text { if } s>\frac{1}{2} .\end{cases}
$$

Reduction to $\Omega_{R}$. Our objective in this section is now to characterize the restriction of $u^{\varepsilon}$ to $\Omega_{R}$ (a domain independent of $\varepsilon$ ) thanks to DtN maps on 
$\Sigma_{\varepsilon}^{-}$and $\Sigma_{\varepsilon}^{+}$. To be able to define these operators, we need to solve the interior problem inside $O_{\varepsilon}$ :

$$
\begin{cases}-\Delta u^{\varepsilon}-\omega^{2} u^{\varepsilon}=0, & \text { in } O_{\varepsilon} \\ \frac{\partial u^{\varepsilon}}{\partial y}=0, & \text { if } y=0 \text { or } \varepsilon\end{cases}
$$

completed by given Dirichlet data on $\Sigma_{\varepsilon}^{-}$and $\Sigma_{\varepsilon}^{+}$. Such a problem is well posed if and only if $\omega^{2}$ is not in the spectrum of the operator $-\Delta$ with domain:

$$
D(-\Delta)=\left\{u \in H^{1}\left(O_{\varepsilon}\right) / \Delta u \in L^{2}\left(O_{\varepsilon}\right), u=0 \text { on } \Sigma_{\varepsilon}, \frac{\partial u}{\partial n}=0 \text { if } y=0 \text { or } \varepsilon\right\},
$$

that is to say: $\quad \omega^{2} \neq\left\{n^{2} \pi^{2} / \varepsilon^{2}+p^{2} \pi^{2} / L^{2} / n \in \mathbb{N}\right.$ and $\left.p \in \mathbb{N}^{*}\right\}$.

If we assume that the slot is not resonant, i.e. that $\omega L$ is not a multiple of $\pi$ - see equation (9) -, it is clear that the condition (21) is satisfied for $\varepsilon$ small enough. Then one can define the Dirichlet to Neumann-map (the normal $n$ is exterior with respect to $\Omega$ )

$$
T^{\varepsilon}(\omega, L):\left.u\right|_{\boldsymbol{\Sigma}_{\varepsilon}} \mapsto-\left.\frac{\partial u^{\varepsilon}}{\partial n}\right|_{\boldsymbol{\Sigma}_{\varepsilon}}
$$

This operator is continuous from $H^{\frac{1}{2}}\left(\boldsymbol{\Sigma}_{\varepsilon}\right)$ to $H^{-\frac{1}{2}}\left(\boldsymbol{\Sigma}_{\varepsilon}\right)$ and can be computed explicitly:

$$
T^{\varepsilon}(\omega, L): \mathbf{u} \longmapsto \mathbf{g} \Longleftrightarrow \mathbf{g}_{n}^{\varepsilon}=M_{n}^{\varepsilon}(\omega, L) \mathbf{u}_{n}^{\varepsilon},
$$

where the matrices $M_{n}^{\varepsilon}(\omega, L)$ are defined by (note that $\left.\sin \omega L \neq 0\right)$ :

$$
M_{0}^{\varepsilon}(\omega, L)=\frac{\omega}{\sin \omega L}\left[\begin{array}{cc}
\cos \omega L & -1 \\
-1 & \cos \omega L
\end{array}\right]
$$

and, for all strictly positive $n$, setting $\xi_{n}^{\varepsilon}(\omega)=\sqrt{\pi^{2} n^{2} / \varepsilon^{2}-\omega^{2}}>0$ :

$$
M_{n}^{\varepsilon}(\omega, L)=\frac{\xi_{n}^{\varepsilon}(\omega)}{\sinh \xi_{n}^{\varepsilon}(\omega) L}\left[\begin{array}{cc}
\cosh \xi_{n}^{\varepsilon}(\omega) L & -1 \\
-1 & \cosh \xi_{n}^{\varepsilon}(\omega) L
\end{array}\right], \forall n \in \mathbb{N}^{*}
$$


It is easy to see that these matrices have the following properties $(\mathbf{u} \cdot \mathbf{v}$ and $|\mathbf{u}|$ denote respectively the usual inner product and associated norm in $\left.\mathbb{C}^{2}\right)$ :

$$
\left\{\begin{array}{l}
\left(M_{0}^{\varepsilon}(\omega, L) \mathbf{u}\right) \cdot \mathbf{u} \in \mathbb{R},\left(M_{0}^{\varepsilon}(\omega, L) \mathbf{u}\right) \cdot \mathbf{v} \leqslant \frac{2 \omega}{\sin \omega L}|\mathbf{u}||\mathbf{v}| \\
\left(M_{n}^{\varepsilon}(\omega, L) \mathbf{u}\right) \cdot \mathbf{u} \in \mathbb{R}^{+},\left(M_{n}^{\varepsilon}(\omega, L) \mathbf{u}\right) \cdot \mathbf{v} \leqslant \frac{2 \xi_{n}^{\varepsilon}(\omega)}{\tanh \xi_{n}^{\varepsilon}(\omega) L}|\mathbf{u}||\mathbf{v}|
\end{array}\right.
$$

By construction, the restriction of $u^{\varepsilon}$ to $\Omega$ is characterized by the transparent condition on $\boldsymbol{\Sigma}_{\varepsilon}$ :

$$
\left.\frac{\partial \mathbf{u}^{\varepsilon}}{\partial n}\right|_{\boldsymbol{\Sigma}_{\varepsilon}}+\left.T^{\varepsilon}(\omega, L) \mathbf{u}^{\varepsilon}\right|_{\boldsymbol{\Sigma}_{\varepsilon}}=0
$$

Equivalently, the restriction of $u^{\varepsilon}$ to $\Omega_{R}$ is characterized as the unique solution of the problem $\left(\varphi_{f} \in H^{1}\left(\Omega_{R}\right)\right.$ is defined by $\left(\varphi_{f}, v\right)_{H^{1}\left(\Omega_{R}\right)}=(f, v)_{L}^{2}\left(\Omega_{R}\right)$ for any $\left.v \in H^{1}\left(\Omega_{R}\right)\right)$

$$
\text { Find } u^{\varepsilon} \in H^{1}\left(\Omega_{R}\right) \text { such that : } \quad A_{R} u^{\varepsilon}+B_{0}^{\varepsilon} u^{\varepsilon}+R^{\varepsilon} u^{\varepsilon}=\varphi_{f} \text {, }
$$

where the operators $A_{R}, B_{0}^{\varepsilon}$ and $R^{\varepsilon}, H^{1}\left(\Omega_{R}\right) \rightarrow H^{1}\left(\Omega_{R}\right)$, are defined, using the Riesz theorem, by :

$$
\left\{\begin{array}{l}
\left(A_{R} u ; v\right)_{H^{1}\left(\Omega_{R}\right)}=\int_{\Omega_{R}}\left(\nabla u \overline{\nabla v}-\omega^{2} u \bar{v}\right)+\sum_{n=-\infty}^{+\infty} \mu_{n}^{R}(\omega) u_{n}^{R} \overline{v_{N}^{R}} \\
\left(B_{0}^{\varepsilon} u ; v\right)_{H^{1}\left(\Omega_{R}\right)}=M_{0}^{\varepsilon} \mathbf{u}_{0}^{\varepsilon} \mathbf{v}_{0}^{\varepsilon}, \quad\left(R^{\varepsilon} u ; v\right)_{H^{1}\left(\Omega_{R}\right)}=\sum_{n=-\infty}^{+\infty} M_{n}^{\varepsilon} \mathbf{u}_{n}^{\varepsilon} \mathbf{v}_{n}^{\varepsilon}
\end{array}\right.
$$

In the same way, $u^{0} \in H^{1}\left(\Omega_{R}\right)$ is the unique solution of : $\quad A_{R} u^{0}=\varphi_{f}$.

\subsection{Convergence of $u^{\varepsilon}$ to $u^{0}$.}

We only prove the $H^{1}$-error estimate of (11). The $L^{2}$-error estimate is shown by a duality argument as in [1]. From (27) and (29), we deduce:

$$
\left(A_{R}+B_{0}^{\varepsilon}+R^{\varepsilon}\right)\left(u^{\varepsilon}-u^{0}\right)=-\left(B_{0}^{\varepsilon}+R^{\varepsilon}\right) u^{0} .
$$

The conclusion follows from the two following lemmas and from (the operator norms below are norms in $\left.L\left(H^{1}\left(\Omega_{R}\right)\right)\right)$ :

$$
\left\|u^{\varepsilon}-u^{0}\right\| \leqslant\left\|\left(A_{R}+B_{0}^{\varepsilon}+R^{\varepsilon}\right)^{-1}\right\|\left(\left\|B_{0}^{\varepsilon} u^{0}\right\|+\left\|R^{\varepsilon} u^{0}\right\|\right)
$$


Lemma 3 If the condition (9) is not satisfied, there exists $\varepsilon_{0}(\omega, R, L)$ strictly positive and $C(\omega, R, L)$ real such that for all $0<\varepsilon<\varepsilon_{0}$ :

$$
\left\|\left(A_{R}+B_{0}^{\varepsilon}+R^{\varepsilon}\right)^{-1}\right\|_{L\left(H^{1}\left(\Omega_{R}\right)\right)} \leqslant C(\omega, R, L) .
$$

Lemma 4 If the condition (9) is not satisfied, there exists $\varepsilon_{0}(\omega, R, L, F)$ strictly positive and $C(\omega, R, L, F)$ real such that for all $0<\varepsilon<\varepsilon_{0}$ :

$$
\left\{\begin{array}{l}
\left\|B_{0}^{\varepsilon} u^{0}\right\|_{H^{1}\left(\Omega_{R}\right)} \leqslant C(\omega, R, L, F) \varepsilon \sqrt{|\log \varepsilon|}\|f\|_{L^{2}(\Omega)}, \\
\left\|R^{\varepsilon} u^{0}\right\|_{H^{1}\left(\Omega_{R}\right)} \leqslant C(\omega, R, L, F) \varepsilon\|f\|_{L^{2}(\Omega)} .
\end{array}\right.
$$

Proof of lemma 3. We make a proof by contradiction. If (32) were false, there would exist a sequence $v^{\varepsilon} \in H^{1}\left(\Omega_{R}\right), \varepsilon \rightarrow 0$, such that:

$$
\left\|v^{\varepsilon}\right\|_{H^{1}\left(\Omega_{R}\right)}=1 \text { and } \varphi^{\varepsilon}=\left(A_{R}+B_{0}^{\varepsilon}+R^{\varepsilon}\right) v^{\varepsilon} \rightarrow 0, \quad \text { in } H^{1}\left(\Omega_{R}\right) .
$$

By compactness, let us extract from $v^{\varepsilon}$ a subsequence (still denoted $v^{\varepsilon}$ ) with $\varepsilon \rightarrow 0$ such that:

$$
v^{\varepsilon} \rightarrow v^{0} \quad \text { weakly in } H^{1}\left(\Omega_{R}\right) \text { and strongly in } L^{2}\left(\Omega_{R}\right) \text {. }
$$

For any $v \in H^{1}\left(\Omega_{R}\right)$, one has:

$$
\left(\varphi^{\varepsilon} ; v\right)_{H^{1}\left(\Omega_{R}\right)}=\left(A_{R} v^{\varepsilon} ; v\right)+b i g\left(B_{0}^{\varepsilon} v^{\varepsilon} ; v\right)+\left(R^{\varepsilon} v^{\varepsilon} ; v\right)
$$

Let us introduce the subspace $H_{00}^{1}\left(\Omega_{R}\right)$ of $H^{1}\left(\Omega_{R}\right)$ made of functions vanishing in a neighborhood $V$ of $A^{-}$and $A^{+}(v=0$ in $V \cap \Omega)$. For any $v \in H_{00}^{1}\left(\Omega_{R}\right)$, as soon as $\varepsilon$ is small enough, $\left(B_{0}^{\varepsilon} \varphi^{\varepsilon} ; v\right)=0$ and $\left(R^{\varepsilon} \varphi^{\varepsilon} ; v\right)=0$. Thus

$$
\left(\varphi^{\varepsilon} ; v\right)_{H^{1}\left(\Omega_{R}\right)}=\left(A_{R} v^{\varepsilon} ; v\right)
$$

Taking the limit as $\varepsilon \rightarrow 0$, we get $\left(A_{R} v^{0} ; v\right)_{H^{1}\left(\Omega_{R}\right)}=0, \forall v \in H_{00}^{1}\left(\Omega_{R}\right)$ which implies, by density of $H_{00}^{1}\left(\Omega_{R}\right)$ in $H^{1}\left(\Omega_{R}\right)$ that $A_{R} v^{0}=0$ that is to say, since $A_{R}$ is an isomorphism (this is nothing but the well-posedness of the slotless problem), $v^{0}=0$.

We choose now $v=v^{\varepsilon}$ in (36), and consider the real part of the resulting 
equality. We obtain, by definition of $A_{R}, B_{0}^{\varepsilon}$ and $R^{\varepsilon}$ :

$$
\left\{\begin{aligned}
\operatorname{Re}\left(\varphi^{\varepsilon} ; v^{\varepsilon}\right)_{H^{1}\left(\Omega_{R}\right)}= & \int_{\Omega_{R}}\left|\nabla v^{\varepsilon}\right|^{2}-\omega^{2} \int_{\Omega_{R}}\left|v^{\varepsilon}\right|^{2}+\operatorname{Re} \sum_{n=0}^{+\infty}\left(\mu_{n}^{R}(\omega)\right)\left|u_{n}^{R}\right|^{2} \\
& +M_{0}^{\varepsilon}(\omega, L) \mathbf{v}_{0}^{\varepsilon} \cdot \mathbf{v}_{0}^{\varepsilon}+\sum_{n=1}^{+\infty} M_{n}^{\varepsilon}(\omega, L) \mathbf{v}_{n}^{\varepsilon} \cdot \mathbf{v}_{n}^{\varepsilon} .
\end{aligned}\right.
$$

Due to properties (6) and (25), we thus have:

$$
\int_{\Omega_{R}}\left|\nabla v^{\varepsilon}\right|^{2} \leqslant\left|\left(\varphi^{\varepsilon} ; v^{\varepsilon}\right)_{H^{1}\left(\Omega_{R}\right)}\right|+\omega^{2} \int_{\Omega_{R}}\left|v^{\varepsilon}\right|^{2}+\frac{2 \omega}{\sin \omega L}\left|\mathbf{v}_{0}^{\varepsilon}\right|^{2} .
$$

Besides, from lemma 2 (with $s=\frac{1}{2}$ ), we get:

$$
\left|\mathbf{v}_{0}^{\varepsilon}\right|^{2} \leqslant C(R) \varepsilon|\log \varepsilon|\left\|v^{\varepsilon}\right\|_{H^{1}\left(\Omega_{R}\right)}^{2}=C(R) \varepsilon|\log \varepsilon|
$$

Hence, from (35), (38), and (39), we deduce that $\nabla v^{\varepsilon}$ converges to 0 in $L^{2}\left(\Omega_{R}\right)$. Therefore $v^{\varepsilon}$ converges strongly to 0 in $H^{1}\left(\Omega_{R}\right)$, which contradicts $(34)$ and concludes the proof.

Proof of lemma 4. As $u^{0}$ is a solution of a Helmholtz problem in $\Omega$ with a source term compactly supported in $F \subset \Omega$, by elliptic regularity there exists a neighborhood $V$ (simply choose $\bar{V} \cap F=\emptyset$ ) of $A^{-}$and $A^{+}$such that $u^{0} \in H^{m}(V \cap \Omega)$, for all $m>0$ and:

$$
\left\|u^{0}\right\|_{H^{m}(V \cap \Omega)} \leqslant C(m, F)\|f\|_{L^{2}(\Omega)}, \quad \forall m>0 .
$$

(i) By definition of $R^{\varepsilon}$ and property (25), we have for all $v \in H^{1}\left(\Omega_{R}\right)$ :

$$
\left(R^{\varepsilon} u^{0} ; v\right)_{H^{1}\left(\Omega_{R}\right)} \leqslant \sum_{n=1}^{+\infty} \frac{2 \xi_{n}^{\varepsilon}(\omega)}{\tanh \xi_{n}^{\varepsilon}(\omega) L}\left|\left(\mathbf{u}^{0}\right)_{n}^{\varepsilon}\right|\left|\mathbf{v}_{n}^{\varepsilon}\right|
$$

For $\varepsilon<\pi / 2 \omega$, we have the inequalities for all $n \in \mathbb{N}^{*}$ :

$$
\frac{2 \xi_{n}^{\varepsilon}(\omega)}{\tanh \left(\xi_{n}^{\varepsilon}(\omega) L\right)} \leqslant \frac{2}{\tanh (\omega L / 2)} \sqrt{1+\frac{\pi^{2} n^{2}}{\varepsilon^{2}}}
$$

The Cauchy-Schwartz inequality leads to:

$$
\left(R^{\varepsilon} u^{0} ; v\right)_{H^{1}\left(\Omega_{R}\right)} \leqslant \frac{2}{\tanh (\omega L / 2)}\left\|\mathbf{u}^{0}\right\|_{H_{*}^{\frac{1}{2}}\left(\boldsymbol{\Sigma}_{\varepsilon}\right)}\|\mathbf{v}\|_{H_{*}^{\frac{1}{2}}\left(\boldsymbol{\Sigma}_{\varepsilon}\right)} .
$$


Thanks to (40) with $m=3$, we can use (18) with $s=2$ for $\mathbf{u}^{0}, s=0$ for $\mathbf{v}$. We get:

$$
\left(R^{\varepsilon} u^{0} ; v\right)_{H^{1}\left(\Omega_{R}\right)} \leqslant C(\omega, R, L, F) \varepsilon\|f\|_{L^{2}(\Omega)}\|v\|_{H^{1}\left(\Omega_{R}\right)} .
$$

The result follows from $\left\|R^{\varepsilon} u^{0}\right\|_{H^{1}\left(\Omega_{R}\right)}=\sup _{v \in H^{1}\left(\Omega_{R}\right)} \frac{\left(R^{\varepsilon} u^{0} ; v\right)_{H^{1}\left(\Omega_{R}\right)}}{\|v\|_{H^{1}\left(\Omega_{R}\right)}}$.

(ii) By definition of $B_{0}^{\varepsilon}$, and due to (25) we have:

$$
\left(B_{0}^{\varepsilon} u^{0} ; v\right)_{H^{1}\left(\Omega_{R}\right)} \leqslant \frac{2 \omega}{\sin (\omega L)}\left|\left(\mathbf{u}^{0}\right)_{0}^{\varepsilon}\right|\left|\mathbf{v}_{0}^{\varepsilon}\right| .
$$

Thanks to (40) with $m=\frac{3}{2}$, we can use (19) with $s=1$ for $\mathbf{u}^{0}, s=\frac{1}{2}$ for $\mathbf{v}$. We get:

$$
\left(B_{0}^{\varepsilon} u^{0} ; v\right)_{H^{1}\left(\Omega_{R}\right)} \leqslant C(\omega, R, L, F) \varepsilon \sqrt{|\log \varepsilon|}\|f\|_{L^{2}(\Omega)}\|v\|_{H^{1}\left(\Omega_{R}\right)} .
$$

One concludes as for point (i).

\section{The case of resonant frequencies: some numerical computations}

Presentation of the experiment. The experiment we simulate is explained in Figure 3. We choose $\omega=2 \pi$, which corresponds to a unit wave length. We shall make our computations with two values of the length of the slot : $\mathrm{L}=0.8$ (the non resonant case) and $\mathrm{L}=1$ (the resonant case). The height $2 H$ of the rectangle $B$ is $2 H=8.0$. The distance from the point source to $B$ is $D=0.5$ in the non resonant case and $D=0.4$ in the resonant case. The numerical results are obtained by a $Q_{2}$ finite element code working with quadrilateral elements [3]. The computational domain (the grey region in figure 3) is delimited by a rectangle of respective dimensions 5 in the $x$ direction and 10 in the $y$ direction. The computational mesh is refined in the neighborhood of the slot as represented in figure 4 (each of the triangle appearing in the picture is divided into three quadrilaterals). In this way, one has about 160 points per wavelength close to the slot and 16 points per wavelength far away. A PML of width 1 , surrounding $\Omega_{c}$, is used to simulate an infinite domain. 
Fig. 3. Presentation of the experiment

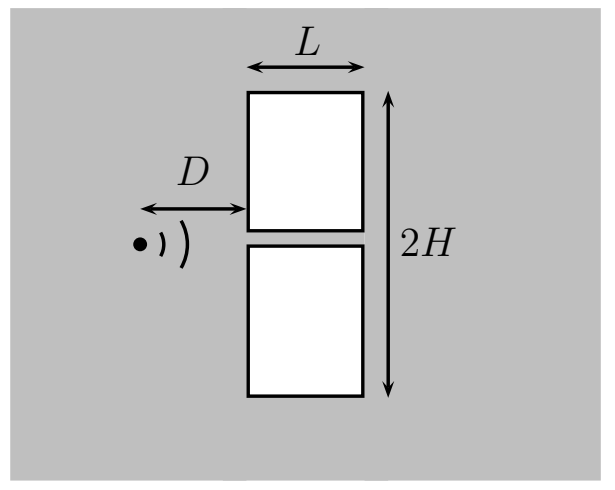

Fig. 4. A computational mesh

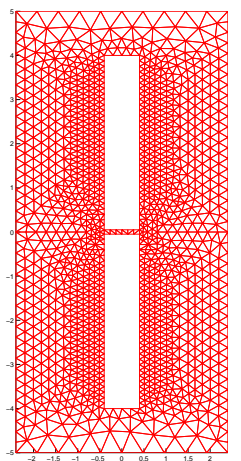

Comments of the results. In figures 5 and 6 , we represent the (real part of) solution $u^{\varepsilon}$ (called total field) in the two cases, for $\varepsilon=10^{-3}$. In this picture, it already appears that much more energy has been transmitted through the slot in the resonant case.

This appears much more clearly when we look at the diffracted fields, namely the difference $u^{\varepsilon}-u^{0}$ between the solution and the solution in the absence of the slot. In figure 7 , we represent these fields for the three values of $\varepsilon=$ $10^{-1}, 10^{-2}, 10^{-3}$. The diffracted field decays much more strongly with $\varepsilon$ in the non resonant case than in the resonant case, for which it is even not so clear that the diffracted field converges to 0 !

In figure 8, we are interested in the solution inside the slot. We represent the variation of the solution along the symmetry line of the slot. Note that the two scales for the two pictures are completely different. Our results show that, while the slot field stabilizes when $\varepsilon$ goes to 0 , it blows up in the resonant case.

We now vary $\varepsilon$ continuously and represent the variations of various norms of the diffracted field and slot fields as a function of $\varepsilon$.

We first look at the results associated to the non resonant case. In figure 9, we represent the $L^{2}$ norm in $\Omega_{c}$ of the diffracted field. The curves confirm the theory: the $L^{2}$ norm of the diffracted field tends to 0 as $O(\varepsilon)$. In figure 10, we represent the variations of the $H^{1}$-norm of the diffracted field as a function of $\varepsilon \sqrt{|\log \varepsilon|}$, the linear behaviour in $\varepsilon \sqrt{|\log \varepsilon|}$ close to 0 seems to confirm the theory, see theorem 1 . In figure 11, we represent the variations of the norm of the solution $u^{\varepsilon}$ in $L^{2}\left(O_{\varepsilon}\right)$. The shape of the curve suggests that this norm decays to 0 sublinearly. When we represent in figure 12 the log of this quantity as a function of $\log \varepsilon$, we obtain a straight line of slope $\sim 0.5$ which confirms a behaviour in $O(\sqrt{\varepsilon})$.

In the resonant case, figure 13 shows that the $L^{2}$ norm of the diffracted field 
Fig. 5. The total field, non resonant
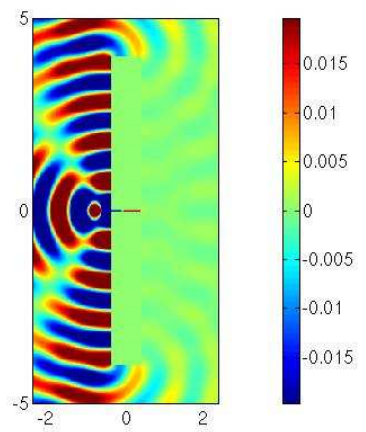

Fig. 7. The diffracted Fields

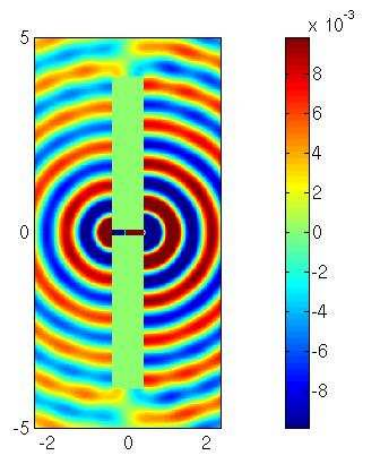

Non resonant, $\varepsilon=10^{-1}$

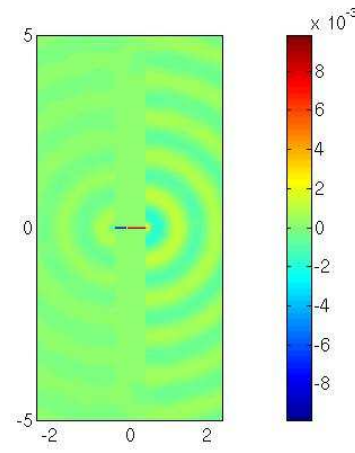

Non resonant, $\varepsilon=10^{-2}$

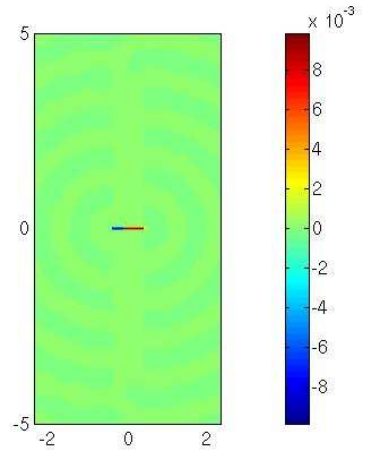

Non resonant, $\varepsilon=10^{-3}$
Fig. 6. The total field, resonant
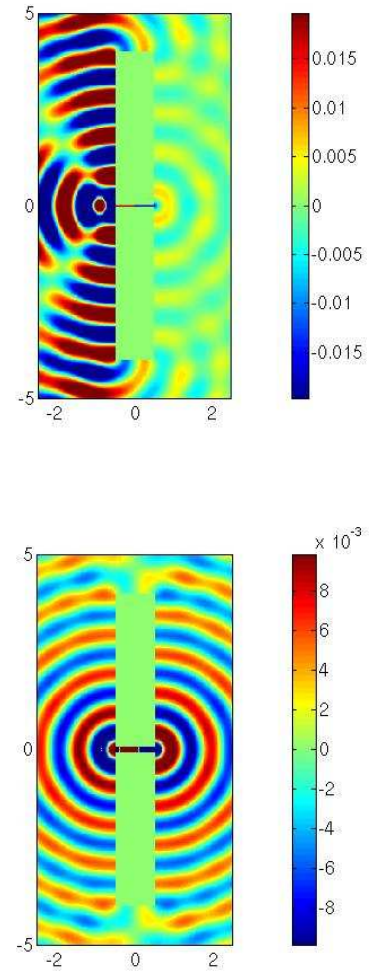

Resonant, $\varepsilon=10^{-1}$

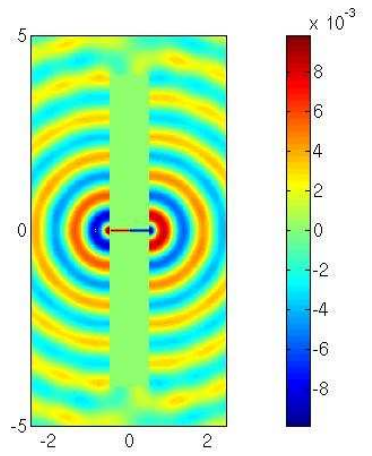

Resonant, $\varepsilon=10^{-2}$

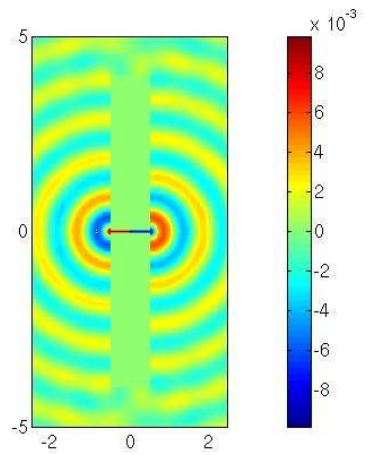

Resonant, $\varepsilon=10^{-3}$ 
Fig. 8. The solution inside the slot for $\varepsilon=10^{-1}, 10^{-2}, 10^{-3}$, left : non resonant case, right: resonant case.

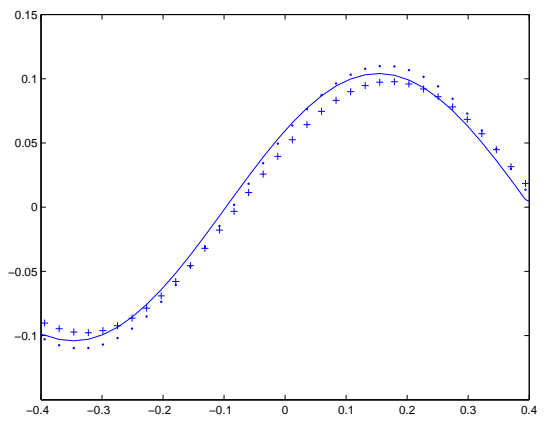

Fig. 9. $\left\|u^{\varepsilon}-u^{0}\right\|_{L^{2}\left(\Omega_{c}\right)}=f(\varepsilon)$

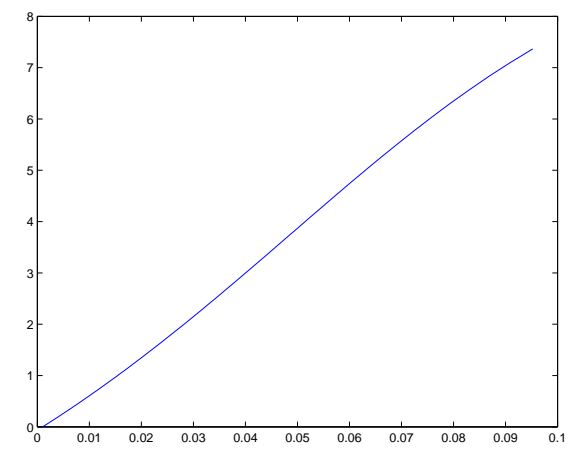

Fig. 11. $\left\|u^{\varepsilon}\right\|_{L^{2}\left(O_{\varepsilon}\right)}=f(\varepsilon)$

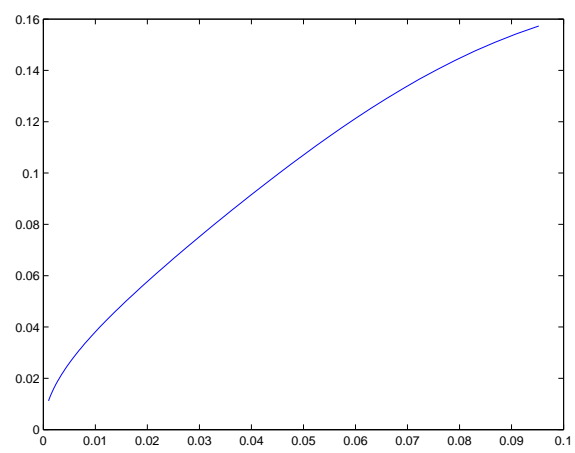

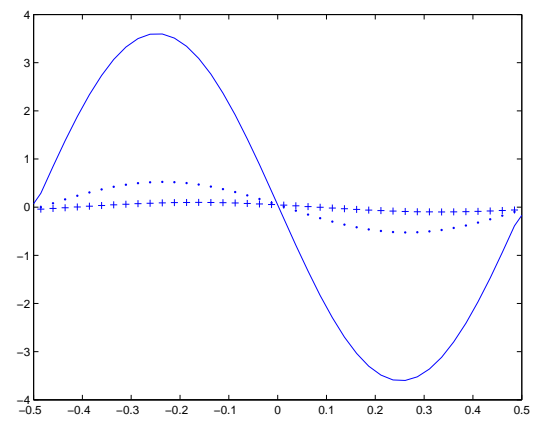

Fig. 10. $\left\|u^{\varepsilon}-u^{0}\right\|_{H^{1}\left(\Omega_{R}\right)}=f\left(\varepsilon \sqrt{\left|\log _{10} \varepsilon\right|}\right)$

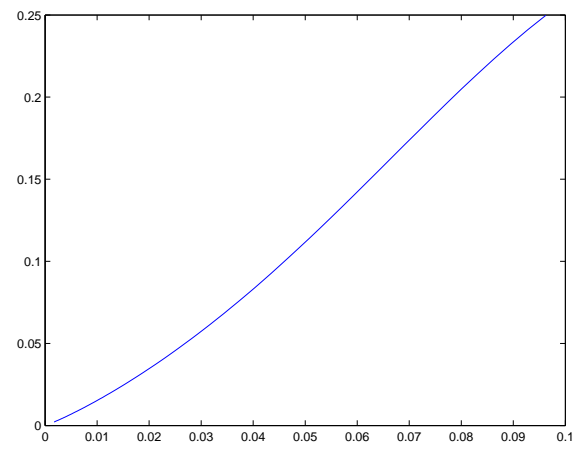

Fig. 12. $\log \left\|u^{\varepsilon}\right\|_{L^{2}\left(O_{\varepsilon}\right)}=f\left(\log _{10} \varepsilon\right)$

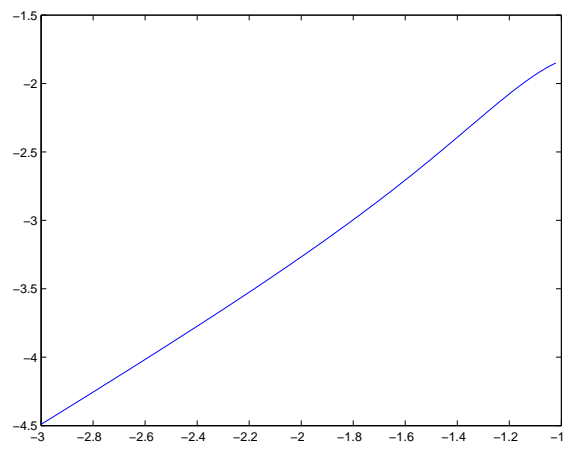

converges to 0 sublinearly. The result of figure 17 (we represent the inverse of the $L^{2}$ norm of the diffracted field as a function of $\left.\log \varepsilon\right)$ suggests in fact that if behaves as $1 /(A \log \varepsilon+B+o(1))$. In figures 15 and 16, we are interested into the $H^{1}$ norm of the diffracted fields. Our results suggest that this norm decays as $1 / \sqrt{(A \log \varepsilon+B+o(1))}$. Finally, figure 17 shows that the $L^{2}$ norm of the slot field blows up when $\varepsilon$ goes to 0 and figure 18 suggests more precisely that it behaves as $1 /(\sqrt{\varepsilon}|\log \varepsilon|)$.

Finally, in figure 19, we fix $\varepsilon=10^{-3}$ and make $\omega$ vary such that the frequency 
Fig. 13. $\left\|u^{\varepsilon}-u^{0}\right\|_{L^{2}\left(\Omega_{c}\right)}=f(\varepsilon)$

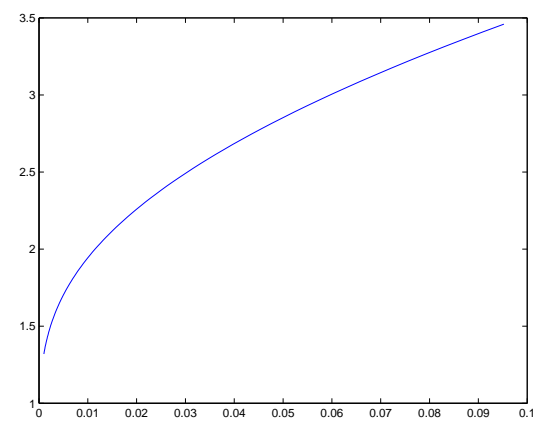

Fig. 15. $\left\|u^{\varepsilon}\right\|_{H^{1}\left(\Omega_{c}\right)}=f(\varepsilon)$

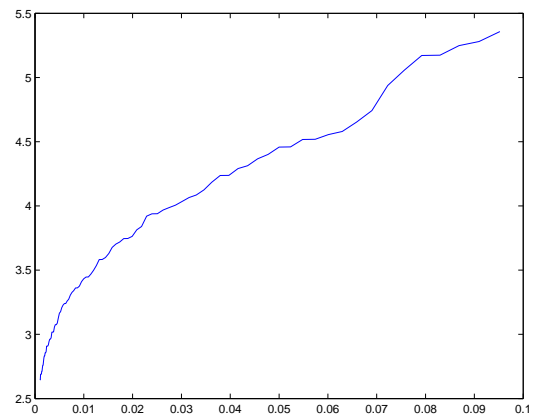

Fig. 17. $\sqrt{\varepsilon} \log \varepsilon\left\|u^{\varepsilon}\right\|_{L^{2}\left(O_{\varepsilon}\right)}=f(\varepsilon)$

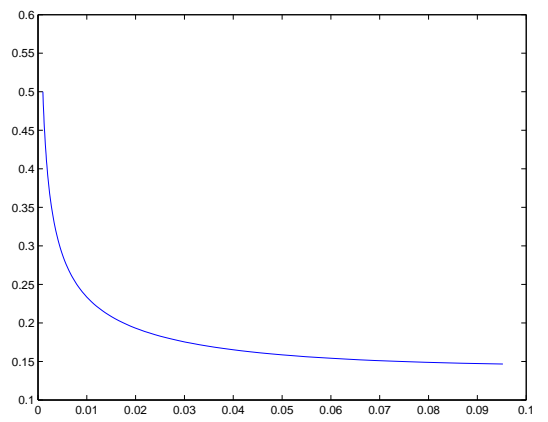

Fig. 14. $1 /\left\|u^{\varepsilon}-u^{0}\right\|_{L^{2}\left(\Omega_{c}\right)}=f(\log \varepsilon)$

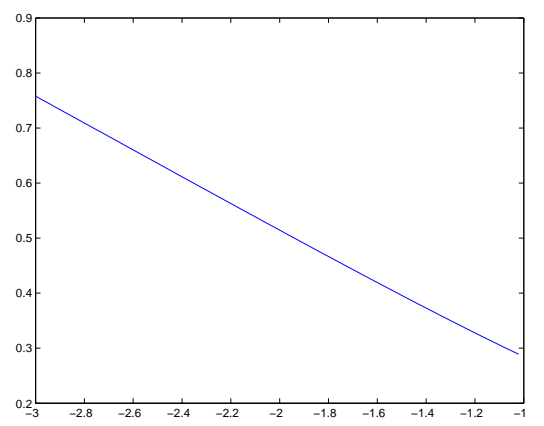

Fig. 16. $\frac{1}{\left\|u^{\varepsilon}\right\|_{H^{1}\left(O_{\varepsilon}\right)}^{2}}=f\left(\log _{10} \varepsilon\right)$

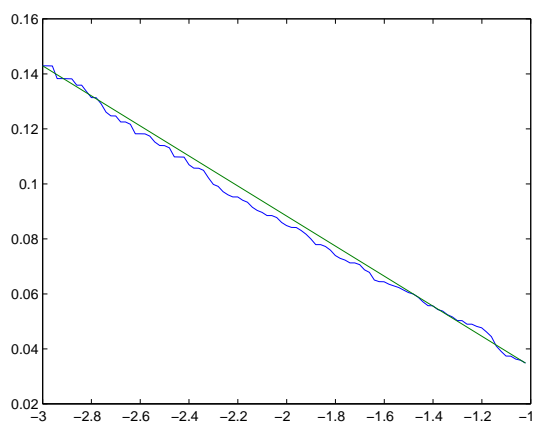

Fig. 18. $\left\|u^{\varepsilon}\right\|_{L^{2}\left(O_{\varepsilon}\right)}=f(\varepsilon)$

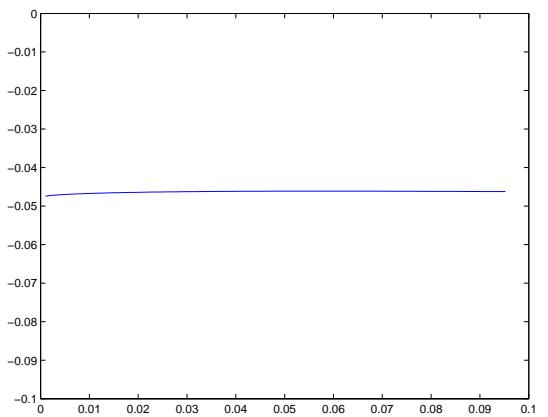

$\omega / 2 \pi$ goes from 0 to 2.5 . One observes that the two curves depicting respectively the $L^{2}$ norm of the diffracted field (normalized by the one of $u^{0}$ ) and the $L^{2}$ norm of the slot field present very sharp peaks corresponding to the resonant frequencies $\left(\omega / 2 \pi\right.$ is an integer multiple of $\left.\frac{1}{2}\right)$. One also observes the the height of these peaks decays with $\omega / 2 \pi$ for sufficiently large $\omega / 2 \pi$. 
Fig. 19. Dependance of the diffracted field with respect to the frequency
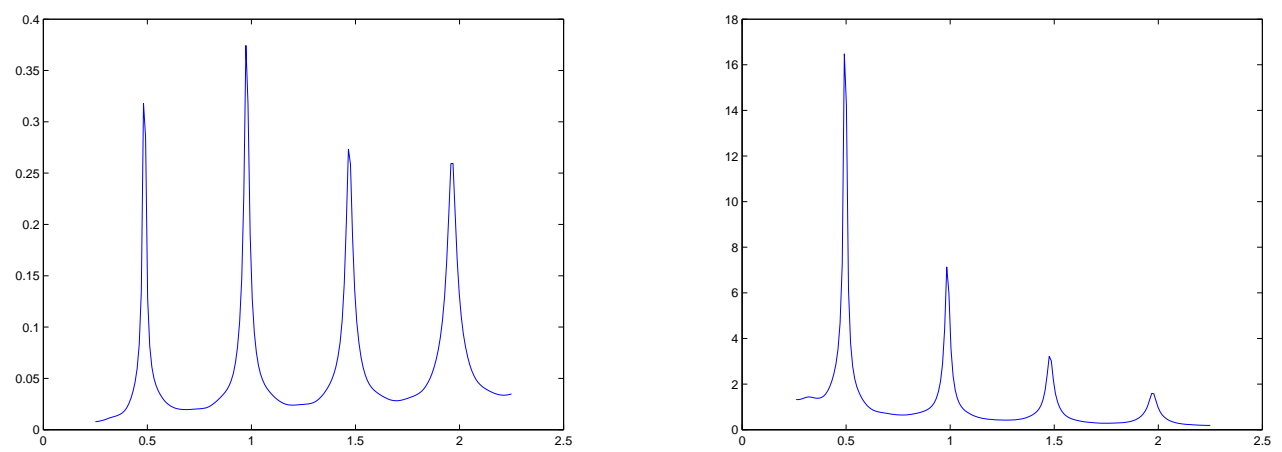

$$
\left\|u^{\varepsilon}-u^{0}\right\|_{L^{2}(\Omega)} /\left\|u^{0}\right\|_{L^{2}(\Omega)}=f(\omega / 2 \pi) \quad\left\|u^{\varepsilon}-u^{0}\right\|_{L^{2}\left(O_{\varepsilon}\right)} /\left\|u^{0}\right\|_{L^{2}\left(O_{\varepsilon}\right)}=f(\omega / 2 \pi)
$$

\section{The case of resonant frequencies: a formal asymptotic analysis}

We now provide a (formal) analysis of the results observed in the previous section thanks to the help of matched asympotic expansions. Of course, it is not our purpose to present in detail this (rather complicated) technique and we refer the reader to the references [4], [5] for more information and to [6] for an application to the semi-infinite slot problem. We shall restrict ourselves to present the main ideas of the method. In particular, we shall introduce our Ansatz without particular justification, despite the fact it can be done (rather tediously) as in [7].

Throughout this section, we assume that:

$$
\omega L=\ell \pi, \quad \ell \in \mathbb{N}^{*} .
$$

\subsection{The Ansatz of matched asymptotic expansions}

The principle is to decompose the domain $\Omega_{\varepsilon}$ into four (overlapping) subdomains which are:

- a "far field" zone (or exterior zone) $\Omega_{F}(\varepsilon)$ in which we shall describe the behaviour of the solution outside the slot.

- a "slot" zone $\Omega_{S}(\varepsilon)$ to describe the behaviour of the solution in the slot.

- two "near field" zones (or junction zones) $\Omega_{N}^{-}(\varepsilon)$ and $\Omega_{N}^{+}(\varepsilon)$ in which we describe the behaviour of the solution at the neighborhood of $\mathbf{A}^{-}$and $\mathbf{A}^{+}$.

The precise definition of these domains uses four functions of $\varepsilon$ :

$$
0<\eta_{F}^{-}(\varepsilon)<\eta_{F}^{+}(\varepsilon), 0<\eta_{S}^{-}(\varepsilon)<\eta_{S}^{+}(\varepsilon)
$$


that are supposed to belong to the class of continuous functions $\eta(\varepsilon)$ satisfying:

$$
\begin{aligned}
& \lim _{\varepsilon \rightarrow 0} \eta(\varepsilon)=0, \quad \lim _{\varepsilon \rightarrow 0} \eta(\varepsilon) / \varepsilon=+\infty \\
& \left\{\begin{array}{l}
\Omega_{F}(\varepsilon)=\left\{\mathbf{x} \in \Omega /\left|\mathbf{x}-\mathbf{A}^{-}\right|>\eta_{F}^{-}(\varepsilon) \text { and }\left|\mathbf{x}-\mathbf{A}^{+}\right|>\eta_{F}^{-}(\varepsilon)\right\} \\
\Omega_{S}(\varepsilon)=\left\{\mathbf{x} \in O_{\varepsilon} / x>\eta_{S}^{-}(\varepsilon) \text { and } L-x>\eta_{S}^{-}(\varepsilon)\right\} \\
\Omega_{N}^{ \pm}(\varepsilon)=\left\{\mathbf{x} \in \Omega /\left|\mathbf{x}-\mathbf{A}^{ \pm}\right|<\eta_{F}^{+}(\varepsilon)\right\} \cup\left\{\mathbf{x} \in O_{\varepsilon} /|x-a \pm|<\eta_{S}^{+}(\varepsilon)\right\}
\end{array}\right.
\end{aligned}
$$

Note in particular that, when $\varepsilon$ tends to $0, \Omega_{N}^{-}(\varepsilon)$ and $\Omega_{N}^{+}(\varepsilon)$ collapse to one point, $\Omega_{S}(\varepsilon)$ to the segment $\left[\mathbf{A}^{-}, \mathbf{A}^{+}\right]$while $\Omega_{F}(\varepsilon)$ converges to $\Omega$.

The next step consists in working with normalized coordinates in each zone and postulating some asymptotic expansion of the solution in these coordinates. For $2 \mathrm{D}$ problems, it is well known that powers of $\varepsilon$ are not sufficient and one must use the set of gauge functions:

$$
f_{i, k}(\varepsilon)=\varepsilon^{i} \log ^{k} \varepsilon, \quad \text { where }(i, k) \text { are integers. }
$$

One of the difficulties is to choose the good set of indices $(i, k)$ in each zone but we shall be guided by our numerical results. In the paragraph below, we present our Ansatz and point out the leading term in each expansion (the one we are interested in in this paper, the other terms being lower order correctors).

Notation. From now on, for any function $v^{\varepsilon}$ defined as a formal series:

$$
v^{\varepsilon}=\sum_{i} \sum_{k} \varepsilon^{i} \log ^{k} \varepsilon v_{i}^{k}
$$

we shall set $v_{i}^{\varepsilon}=\sum_{k} \log ^{k} \varepsilon v_{i}^{k}$.

This allows us to distinguish the dependence of $v^{\varepsilon}$ with respect to $\varepsilon$ and $\log \varepsilon$, which is justified by the fact that:

$$
\forall k \in \mathbb{Z}, \quad \varepsilon=o\left(|\log \varepsilon|^{k}\right) .
$$

Solution in the far field zone. We keep here the physical coordinates $(x, y)$ and look for $u^{\varepsilon}$ of the form:

$$
\left\{\begin{aligned}
u^{\varepsilon}(x, y) & =\sum_{i=0}^{+\infty} \sum_{k=-\infty}^{i} \varepsilon^{i} \log ^{k} \varepsilon u_{i}^{k}(x, y)+o\left(\varepsilon^{\infty}\right) . \\
& =\sum_{i=0}^{+\infty} \varepsilon^{i} u_{i}^{\varepsilon}(x, y)+o\left(\varepsilon^{\infty}\right)=u_{0}^{\varepsilon}+o(1)
\end{aligned}\right.
$$


The leading term corresponds to $(i, k)=(0,0)$ which means that $u^{\varepsilon}$ converges to a limit in $\Omega$. In the expansion below, the functions $u_{i}^{k}$ and $u_{i}^{\varepsilon}$ are defined in the canonical (i.e. independent of $\varepsilon$ ) slotless domain $\Omega$. Inserting (54) into $(4)$, it is easy to see that:

$$
\Delta u_{0}^{\varepsilon}+\omega^{2} u_{0}^{\varepsilon}=-f, \quad \text { in } \Omega, \quad \frac{\partial u_{0}^{\varepsilon}}{\partial n}=0, \quad \text { on } \partial \Omega \backslash\left\{A^{-}, A^{+}\right\} .
$$

The fact that $u_{0}^{\varepsilon}$ satisfies the Neumann condition except at points $A^{-}$and $A^{+}$allows $u_{0}^{\varepsilon}$ to be singular at $A^{-}$and $A^{+}$. Therefore, one needs additional informations about the behaviour of $u_{0}^{\varepsilon}$ in the neighborhood of these two points.

Solution in the slot zone. We use the coordinates $(x, y / \varepsilon)$ that maps $O_{\varepsilon}$ into $O_{1}$, a slot of unit width, and look for $u^{\varepsilon}$ of the form:

$$
\left\{\begin{aligned}
u^{\varepsilon}(x, y) & =U^{\varepsilon}\left(x, \frac{y}{\varepsilon}\right)=\sum_{i=-1}^{+\infty} \sum_{k=-\infty}^{i} \varepsilon^{i} \log ^{k} \varepsilon U_{i}^{k}\left(x, \frac{y}{\varepsilon}\right)+o\left(\varepsilon^{\infty}\right) \\
& =\sum_{i=-1}^{+\infty} \varepsilon^{i} U_{i}^{\varepsilon}\left(x, \frac{y}{\varepsilon}\right)=\frac{1}{\varepsilon} U_{-1}^{\varepsilon}(x, y / \varepsilon)+U_{0}^{\varepsilon}(x, y / \varepsilon)+o(1) .
\end{aligned}\right.
$$

The leading term corresponds this time to $(i, k)=(-1,-1)$ which means that $u^{\varepsilon}$ blows up in $O_{\varepsilon}$ as $O(1 /(\varepsilon \log \varepsilon))$. The first term $U_{-1}^{\varepsilon}$ and the next term $U_{0}^{\varepsilon}$ will play a role in the calculations. Substituting (56) into (4), one sees that $U_{i}^{\varepsilon}$ depends only from $x$ and that:

$$
\frac{d^{2} U_{i}^{\varepsilon}}{d x^{2}}+\omega^{2} U_{i}^{\varepsilon}=0, \quad \text { in } O_{1}
$$

Note that, to determine $U_{i}^{\varepsilon}$, one needs additional informations at $x=0$ or $L$.

Solution in the near field zones. We present only the case of the zone $\Omega_{N}^{-}(\varepsilon)$, the case of the zone $\Omega_{N}^{+}(\varepsilon)$ is obviously similar. We use the coordinates $(x / \varepsilon, y / \varepsilon)$, a scaling that maps the region $\Omega_{N}^{-}(\varepsilon)$ in to a domain that converges to an infinite "T-shape" domain $\widehat{\Omega}_{N}^{-}$defined as:

$$
\widehat{\Omega}_{N}^{-}=\left\{(X, Y) \in \mathbb{R}^{2} / X \leqslant 0\right\} \cup\left\{(X, Y) \in \mathbb{R}^{2} / X \geqslant 0 \text { et } 0 \leqslant Y \leqslant 1\right\} .
$$

We look for $u^{\varepsilon}$ of the form (we point out in the second line the terms that will play a role in the computations):

$$
\left\{\begin{aligned}
u^{\varepsilon}(x, y) & =\mathbf{U}^{-, \varepsilon}\left(\frac{x}{\varepsilon}, \frac{y}{\varepsilon}\right)=\sum_{i=0}^{+\infty} \sum_{k=-\infty}^{i} \varepsilon^{i} \log ^{k} \varepsilon \mathbf{U}_{i}^{-, k}\left(\frac{x}{\varepsilon}, \frac{y}{\varepsilon}\right)+o\left(\varepsilon^{\infty}\right) \\
& =\sum_{i=0}^{+\infty} \varepsilon^{i} \mathbf{U}_{i}^{-, \varepsilon}\left(\frac{x}{\varepsilon}, \frac{y}{\varepsilon}\right)+o\left(\varepsilon^{\infty}\right)=\mathbf{U}_{0}^{-, \varepsilon}\left(\frac{x}{\varepsilon}, \frac{y}{\varepsilon}\right)+o(1)
\end{aligned}\right.
$$


where the $\mathbf{U}_{i}^{-, k}$ and $\mathbf{U}_{i}^{-, \varepsilon}$ are defined in $\widehat{\Omega}_{N}^{-}$. Note that this time, it is not clear from the numerical computations how to determine the good leading term of the expansion. Substituting (59) into (4), one easily obtains:

$$
\Delta \mathbf{U}_{0}^{-, \varepsilon}=0, \quad \text { in } \widehat{\Omega}_{N}^{-}, \quad \frac{\partial \mathbf{U}_{0}^{-, \varepsilon}}{\partial n}=0, \quad \text { on } \partial \widehat{\Omega}_{N}^{-}
$$

To determine $\mathbf{U}_{0}^{-, \varepsilon}$, one needs to prescribe its behaviour at infinity in $\widehat{\Omega}_{N}^{-}$.

In the same way, to describe the near field in the second junction region using the scaled coordinates $(X=(x-L) / \varepsilon, Y=y / \varepsilon)$, we introduce an harmonic function $\mathbf{U}_{0}^{+, \varepsilon}$ that is defined in the domain:

$$
\widehat{\Omega}_{N}^{+}=\left\{(X, Y) \in \mathbb{R}^{2} / X \geqslant 0\right\} \cup\left\{(X, Y) \in \mathbb{R}^{2} / X \leqslant 0 \text { et } 0 \leqslant Y \leqslant 1\right\} .
$$

and satisfies the homogeneous Neumann condition on $\partial \widehat{\Omega}_{N}^{+}$.

Summary of the first step of the method. Our objective will be to determine the four functions $u_{0}^{\varepsilon}, U_{-1}^{\varepsilon}, \mathbf{U}_{0}^{-, \varepsilon}$ and $\mathbf{U}_{0}^{+, \varepsilon}$. We know the PDE's satisfied by these functions in their respective domains of definition, as well as the boundary conditions they satisfy. We still need:

- to know the asymptotic behaviour of $u_{0}^{\varepsilon}$ at the neighborhood of $A^{-}$and $A^{+}$, - to know the boundary conditions for $U_{-1}^{\varepsilon}$ at $x=0$ and $x=L$,

- to know the asymptotic behaviour at infinity of $\mathbf{U}_{0}^{-, \varepsilon}$ and $\mathbf{U}_{0}^{+, \varepsilon}$.

This missing information will be provided by the application of the matching principle which will express, in some approximate way, that the various expansions (54), (56), (59) represent the same function in the overlapping zones.

Remark. Although we do not need to determine $U_{0}^{\varepsilon}$, this function will naturally appear in the application of the matching principle.

Matching between the near field and the slot field. On the domain $\Omega_{S}(\varepsilon) \cap \Omega_{N}^{-}(\varepsilon), U^{\varepsilon}(x, y / \varepsilon)$ and $\mathbf{U}^{\varepsilon}(x / \varepsilon, y / \varepsilon)$ must coincide. In particular, we need to match the truncated expansions ( in $o(1)$ ) in (56) and (59). Noticing that, in the overlapping zone, $x \rightarrow 0$ and $x / \varepsilon \rightarrow+\infty$, this can be reduced to:

$$
\lim _{x \rightarrow 0, \frac{x}{\varepsilon} \rightarrow+\infty}\left[\frac{1}{\varepsilon} U_{-1}^{\varepsilon}\left(x, \frac{y}{\varepsilon}\right)+U_{0}^{\varepsilon}\left(x, \frac{y}{\varepsilon}\right)-\mathbf{U}_{0}^{-, \varepsilon}\left(\frac{x}{\varepsilon}, \frac{y}{\varepsilon}\right)\right]=0 .
$$

In the domain $\left[0 ;+\infty\left[{ }_{X} \times[0 ; 1]_{Y}\right.\right.$, since the near field $\mathbf{U}_{0}^{-, \varepsilon}$ satisfies the Laplace equation with Neumann boundary condition on the two horizontal boundaries, by separation of variable, if we exclude exponentially growing solutions inside the slot (which can be proved), we know that $\mathbf{U}_{0}^{-, \varepsilon}$ is of the form $\left(\mu_{-}^{\varepsilon}, \nu_{-}^{\varepsilon}\right.$ and 
$\left(\nu_{-}^{\varepsilon}\right)^{p}$ are complex functions of $\left.\log \varepsilon\right)$ :

$$
\mathbf{U}_{0}^{-, \varepsilon}(X, Y)=\mu_{-}^{\varepsilon} X+\nu_{-}^{\varepsilon}+\sum_{p=1}^{+\infty}\left(\nu_{-}^{\varepsilon}\right)^{p} \exp -p \pi X \cos p \pi Y, X>0
$$

Since the slot field satisfies the 1D Helmholtz equation, there exists complex functions $\left(A_{i}^{\varepsilon}, B_{i}^{\varepsilon}, i=-1,0\right)$ of $\log \varepsilon$ such that:

$$
U_{i}^{\varepsilon}(x)=A_{i}^{\varepsilon} \cos \omega x+B_{i}^{\varepsilon} \sin \omega x, \quad i=-1,0 .
$$

Hence, when $x \rightarrow 0$ and $x / \varepsilon \rightarrow+\infty$, one has:

$$
\begin{aligned}
& \frac{1}{\varepsilon} U_{-1}^{\varepsilon}\left(x, \frac{y}{\varepsilon}\right)+U_{0}^{\varepsilon}\left(x, \frac{y}{\varepsilon}\right)=\frac{1}{\varepsilon} A_{-1}^{\varepsilon}+\omega B_{-1}^{\varepsilon} \frac{x}{\varepsilon}+A_{0}^{\varepsilon}+o(1), \\
& \mathbf{U}_{0}^{-, \varepsilon}\left(\frac{x}{\varepsilon}, \frac{y}{\varepsilon}\right)=\mu_{-}^{\varepsilon} \frac{x}{\varepsilon}+\nu_{-}^{\varepsilon}+o(1) .
\end{aligned}
$$

Therefore, (62) leads to: $A_{-1}^{\varepsilon}=0, \quad \omega B_{-1}^{\varepsilon}=\mu_{-}^{\varepsilon}, \quad A_{0}^{\varepsilon}=\nu_{-}^{\varepsilon}$.

By symmetry, we have also:

$$
\mathbf{U}_{0}^{+, \varepsilon}(X, Y)=\mu_{+}^{\varepsilon} X+\nu_{+}^{\varepsilon}+o(1), \quad \text { when } X \rightarrow-\infty .
$$

with the relations: $A_{-1}^{\varepsilon}=0, \quad(-1)^{\ell} \omega B_{-1}^{\varepsilon}=\mu_{+}^{\varepsilon}, \quad(-1)^{\ell} A_{0}^{\varepsilon}=\nu_{+}^{\varepsilon}$.

Matching between the far field and the near field On the domain $\Omega_{F}(\varepsilon) \cap \Omega_{N}^{-}(\varepsilon), u^{\varepsilon}(x, y)$ and $\mathbf{U}^{-,} \varepsilon(x / \varepsilon, y / \varepsilon)$ must coincide. In particular, we need to match the truncated expansions ( in $o(1)$ ) of (54) and (59). Let us use the polar coordinates $\left(r^{-}, \theta^{-}\right)$associated to the point $\mathbf{A}^{-}$(and such that $\theta^{-}=0$ or $\pi$ coincides with the line $\left.x=0\right)$. Noticing that, in the overlapping zone, $r^{-} \rightarrow 0$ and $r^{-} / \varepsilon \rightarrow+\infty$, this can be reduced to:

$$
\lim _{r^{-} \rightarrow 0, \frac{r^{-}}{\varepsilon} \rightarrow+\infty}\left[u_{0}^{\varepsilon}\left(r^{-}, \theta^{-}\right)-\mathbf{U}_{0}^{-, \varepsilon}\left(\frac{r^{-}}{\varepsilon}, \theta^{-}\right)\right]=0 .
$$

As $f$ has a compact support include in $\Omega$, there exists $0<R<\inf (H, L)$, such that, for $r^{-}<R$, by separation of variables, $u_{0}^{\varepsilon}$ can be written as a linear combination of:

$$
\begin{cases}J_{p}\left(\omega r^{-}\right) \cos \left(p \theta^{-}\right), & p \in \mathbb{N} \quad\left(\text { smooth functions of } r^{-}\right) \\ Y_{p}\left(\omega r^{-}\right) \cos \left(p \theta^{-}\right), & p \in \mathbb{N} \quad\left(\text { which are singular close to } r^{-}=0\right)\end{cases}
$$


It can be shown (but the proof is omitted here) that the only function $Y_{p}\left(\omega r^{-}\right)$ that may appear in the expansion corresponds to $p=0$. Therefore, we have an expression of the form (for $r^{-}<R$ ):

$$
u_{0}^{\varepsilon}\left(r^{-}, \theta^{-}\right)=\sum_{p=1}^{+\infty}\left(b_{-}^{\varepsilon}\right)^{p} J_{p}\left(\omega r^{-}\right) \cos \left(p \theta^{-}\right)+a_{-}^{\varepsilon} Y_{0}\left(\omega r^{-}\right)+b_{-}^{\varepsilon} J_{0}\left(\omega r^{-}\right) .
$$

In the same way, if we use $\left(\rho^{-}, \theta^{-}\right)$the polar coordinates associated to the variables $(X, Y)$ in $\widehat{\Omega}_{N}^{-}$, since $\mathbf{U}_{0}^{\varepsilon}$ satisfies the homogeneous Laplace equation with homogeneous Neumann condition, by separation of variables, in the domain $\left.\rho^{-}>1\right), \mathbf{U}_{0}^{-, \varepsilon}$ can be written as a linear combination of:

$$
\left\{\left(\rho^{-}\right)^{p} \cos \left(p \theta^{-}\right),\left(\rho^{-}\right)^{-p} \cos \left(p \theta^{-}\right), p \geq 1\right\}, \log \rho^{-}, 1 .
$$

One can show (once again, the proof is omitted here) that $\mathbf{U}_{0}^{-, \varepsilon}$ cannot grow more rapidly than $\log \rho^{-}$when $\rho^{-}$goes to $+\infty$. Therefore, we have an expression of the form $\left(\rho^{-}>1\right)$ :

$$
\mathbf{U}_{0}^{-, \varepsilon}\left(\rho^{-}, \theta^{-}\right)=\sum_{p=1}^{+\infty}\left(\beta_{-}^{\varepsilon}\right)^{p}\left(\rho^{-}\right)^{-p} \cos \left(p \theta^{-}\right)+\alpha_{-}^{\varepsilon} \log \rho^{-}+\beta_{-}^{\varepsilon} .
$$

Recalling that ( $\gamma$ denotes the Euler number [8]):

$$
\lim _{z \rightarrow 0} J_{0}(z)=1, \quad \lim _{z \rightarrow 0} J_{p}(z)=0 \text { for } p \geq 1, \quad Y_{0}(z)=\frac{2}{\pi}\left(\log \left(\frac{z}{2}\right)+\gamma\right)+o(1),
$$

we deduce that:

$$
\left\{\begin{array}{l}
u_{0}^{\varepsilon}\left(r^{-}, \theta^{-}\right)=\frac{2}{\pi} a_{-}^{\varepsilon}\left(\log \frac{\omega r^{-}}{2}+\gamma\right)+b_{-}^{\varepsilon}+o(1), \quad\left(r^{-} \rightarrow 0\right), \\
\mathbf{U}_{0}^{-, \varepsilon}\left(r^{-} / \varepsilon, \theta^{-}\right)=\alpha_{-}^{\varepsilon} \log \frac{r^{-}}{\varepsilon}+\beta_{-}^{\varepsilon}+o(1) \quad\left(r^{-} / \varepsilon \rightarrow+\infty\right) .
\end{array}\right.
$$

Therefore, (70) leads to :

$$
\frac{2}{\pi} a_{-}^{\varepsilon}=\alpha_{-}^{\varepsilon}, \quad \frac{2}{\pi} a_{-}^{\varepsilon}\left(\log \frac{\omega}{2}+\gamma\right)+b_{-}^{\varepsilon}=-\alpha_{-}^{\varepsilon} \log \varepsilon+\beta_{-}^{\varepsilon} .
$$

In the same way (with obvious notation for polar coordinates), we have:

$$
\left\{\begin{array}{l}
u_{0}^{\varepsilon}\left(r^{+}, \theta^{+}\right)=\frac{2}{\pi} a_{+}^{\varepsilon}\left(\log \frac{\omega r^{+}}{2}+\gamma\right)+b_{+}^{\varepsilon}+o(1), \quad\left(r^{+} \rightarrow 0\right), \\
\mathbf{U}_{0}^{+, \varepsilon}\left(\frac{r^{+}}{\varepsilon}, \theta^{+}\right)=\alpha_{+}^{\varepsilon} \log \frac{r^{+}}{\varepsilon}+\beta_{+}^{\varepsilon}+o(1) \quad\left(r^{+} / \varepsilon \rightarrow+\infty\right) .
\end{array}\right.
$$


with the relations:

$$
\frac{2}{\pi} a_{+}^{\varepsilon}=\alpha_{+}^{\varepsilon}, \quad \frac{2}{\pi} a_{+}^{\varepsilon}\left(\log \frac{\omega}{2}+\gamma\right)+b_{+}^{\varepsilon}=-\alpha_{+}^{\varepsilon} \log \varepsilon+\beta_{+}^{\varepsilon} .
$$

We have now all the necessary information to determine the fields $u_{0}^{\varepsilon}, U_{-1}^{\varepsilon}$, $\mathbf{U}_{0}^{-, \varepsilon}$ and $\mathbf{U}_{0}^{+, \varepsilon}$.

\subsection{Determination of the leading terms of the expansion.}

The analysis of the far field $u_{0}^{\varepsilon}$ will rely on the following lemma. The proof, omitted here, can be adapted from those in [7].

Lemma 5 There exists a unique outgoing solution $u^{*}$ of the homogeneous Helmholtz equation in $\Omega$ satisfying the homogeneous Neumann boundary condition on $\partial \Omega \backslash\left\{\mathbf{A}^{-}, \mathbf{A}^{+}\right\}$such that there exists $b^{*} \in \mathbb{C}$ with:

$$
\begin{cases}u^{*}\left(r^{-}, \theta^{-}\right)-\frac{2}{\pi}\left(\log \frac{\omega r^{-}}{2}+\gamma\right) \rightarrow b^{*}, & \text { when } r^{-} \rightarrow 0 \\ u^{*}\left(r^{+}, \theta^{+}\right)+(-1)^{\ell} \frac{2}{\pi}\left(\log \frac{\omega r^{+}}{2}+\gamma\right) \rightarrow(-1)^{\ell+1} b^{*}, & \text { when } r^{+} \rightarrow 0\end{cases}
$$

By linearity, we deduce the following

Corollary 6 If $v$ is an outgoing solution of the homogeneous Helmholtz equation in $\Omega$ with Neumann boundary condition in $\partial \Omega \backslash\left\{\mathbf{A}^{-}, \mathbf{A}^{+}\right\}$and satisfies the local behaviours:

$$
\begin{cases}v\left(r^{-}, \theta^{-}\right)-\frac{2}{\pi} a\left(\log \frac{\omega r^{-}}{2}+\gamma\right) & \text { has a finite limit when } r^{-} \rightarrow 0 \\ v\left(r^{+}, \theta^{+}\right)+(-1)^{\ell} \frac{2}{\pi} a\left(\log \frac{\omega r^{+}}{2}+\gamma\right) & \text { has a finite limit when } r^{+} \rightarrow 0\end{cases}
$$

then, one has: $\quad v=a u^{*}$.

We first notice that, if we integrate the equation (60) over the domain:

$$
\widehat{\Omega}_{N, 1}^{-}=\left\{\mathbf{x} \in \widehat{\Omega}_{N}^{-} / 0<\rho^{+}<1,0<\theta^{+}<\pi\right\} .
$$

One easily obtain the relation: $\quad \alpha_{-}^{\varepsilon}=-\frac{1}{\pi} \mu_{-}^{\varepsilon}$.

In the same way, we show that: $\alpha_{+}^{\varepsilon}=\frac{1}{\pi} \mu_{+}^{\varepsilon}$. 
Therefore, using (75), (67) and (80) (respectively (77), (69) and (81)), one can obtain the relations:

$$
\left\{\begin{array}{l}
\frac{2}{\pi} a_{-}^{\varepsilon}=\alpha_{-}^{\varepsilon}=-\frac{1}{\pi} \mu_{-}^{\varepsilon}=-\frac{\omega B_{-1}^{\varepsilon}}{\pi} \\
\frac{2}{\pi} a_{+}^{\varepsilon}=\alpha_{+}^{\varepsilon}=\frac{1}{\pi} \mu_{+}^{\varepsilon}=(-1)^{\ell} \frac{\omega B_{-1}^{\varepsilon}}{\pi}
\end{array}\right.
$$

which implies in particular: $a_{-}^{\varepsilon}=(-1)^{\ell+1} a_{+}^{\varepsilon} \quad\left(:=a^{\varepsilon}\right)$.

Applying corollary 6 to $v=u^{\varepsilon}-u^{0}$, we obtain: $u_{0}^{\varepsilon}=u^{0}+a^{\varepsilon} u^{*}$.

Therefore $u_{0}^{\varepsilon}$ has the following behaviour at the neighborhood of $A^{+}$and $A^{-}$:

$$
\left\{\begin{array}{l}
u_{0}^{\varepsilon}\left(r^{-}, \theta^{-}\right)=u^{0}\left(A^{-}\right)+a^{\varepsilon}\left[\frac{2}{\pi}\left(\log \frac{\omega r^{-}}{2}+\gamma\right)+b^{*}\right]+o(1) \\
u_{0}^{\varepsilon}\left(r^{+}, \theta^{+}\right)=u^{0}\left(A^{+}\right)+(-1)^{\ell+1} a^{\varepsilon}\left[\frac{2}{\pi}\left(\log \frac{\omega r^{+}}{2}+\gamma\right)+b^{*}\right]+o(1)
\end{array}\right.
$$

Comparing (74,76) and (85), we have:

$$
b_{-}^{\varepsilon}=a^{\varepsilon} b^{*}+u^{0}\left(A^{-}\right), \quad b_{+}^{\varepsilon}=(-1)^{\ell+1} a^{\varepsilon} b^{*}+u^{0}\left(A^{+}\right) .
$$

For the near field we shall use the following result (adapting the proof of [9]):

Lemma 7 There exists a unique function $U_{ \pm}^{*}$, harmonic in $\widehat{\Omega}_{N}^{ \pm}$, which satisfies, when $\rho^{ \pm} \rightarrow+\infty$ and $\mp X \rightarrow+\infty$ :

$$
\mathbf{U}_{ \pm}^{*}\left(\rho^{ \pm}, \theta^{ \pm}\right)=\log \rho^{ \pm}+o(1), \quad \mathbf{U}_{ \pm}^{*}(X, Y)=O(|X|) .
$$

as well as the homogeneous Neumann boundary condition on $\partial \widehat{\Omega}_{N}^{ \pm}$. Moreover, there exists $\nu^{*} \in \mathbb{C}$ such that:

$$
\mathbf{U}_{ \pm}^{*}(X, Y)= \pm \pi X+\nu^{*}+o(1), \quad \text { when } \mp X \rightarrow+\infty
$$

Corollary 8 Let $V_{ \pm}$a solution of the homogeneous Laplace equation in $\widehat{\Omega}_{N}^{ \pm}$ satisfying the homogeneous Neumann boundary condition on $\partial \widehat{\Omega}_{N}^{ \pm}$and:

$$
\begin{cases}V_{ \pm}\left(\rho^{ \pm}, \theta^{ \pm}\right)=\alpha_{ \pm} \log \rho^{ \pm}+\beta_{ \pm}+o(1), & \rho^{ \pm} \rightarrow+\infty \\ V_{ \pm}(X, Y)=O(|X|), & \mp X \rightarrow+\infty\end{cases}
$$

then: $\quad V_{ \pm}=\alpha_{ \pm} \mathbf{U}_{ \pm}^{*}+\beta_{ \pm}$. 
Applying corollary 8 to $\mathbf{U}_{0}^{-, \varepsilon}$ and $\mathbf{U}_{0}^{+, \varepsilon}$, we obtain using equations $(74,76)$, $(75,77)$ and $(86)$ :

$$
\left\{\begin{array}{l}
\mathbf{U}_{0}^{-, \varepsilon}=a^{\varepsilon}\left[\frac{2}{\pi}\left(\mathbf{U}_{-}^{*}+\log \frac{\omega \varepsilon}{2}+\gamma\right)+b^{*}\right]+u^{0}\left(\mathbf{A}^{-}\right) \\
\mathbf{U}_{0}^{+, \varepsilon}=(-1)^{\ell+1} a^{\varepsilon}\left[\frac{2}{\pi}\left(\mathbf{U}_{+}^{*}+\log \frac{\omega \varepsilon}{2}+\gamma\right)+b^{*}\right]+u^{0}\left(\mathbf{A}^{+}\right) .
\end{array}\right.
$$

Moreover, $U_{-1}^{\varepsilon}$ is given via (64) and (83): $\quad U_{-1}^{\varepsilon}(x)=\frac{2}{\omega} a^{\varepsilon} \sin \omega x$.

As can be seen in the formulas (84), (90), (91), we know $u_{0}^{\varepsilon}, \mathbf{U}_{0}^{ \pm, \varepsilon}$ and $U_{-1}^{\varepsilon}$ as soon as we know $a^{\varepsilon}$. To obtain $a^{\varepsilon}$, we have to ensure:

From equations (67) and (69) we deduce: $\quad \nu_{-}^{\varepsilon}=(-1)^{\ell} \nu_{+}^{\varepsilon}$

Equations (90) and (88) lead to:

$$
\left\{\begin{aligned}
\mathbf{U}_{0}^{-, \varepsilon}(X, Y)= & a^{\varepsilon}\left[-2 X+\frac{2}{\pi}\left(\log \frac{\omega \varepsilon}{2}+\gamma+\nu^{*}\right)+b^{*}\right] \\
& +u^{0}\left(\mathbf{A}^{-}\right)+o(1), \\
\mathbf{U}_{0}^{+, \varepsilon}(X, Y)= & (-1)^{\ell+1} a^{\varepsilon}\left[\left(2 X+\frac{2}{\pi}\left(\log \frac{\omega \varepsilon}{2}+\gamma+\nu^{*}\right)\right)+b^{*}\right] \\
& +u^{0}\left(\mathbf{A}^{+}\right)+o(1) .
\end{aligned}\right.
$$

Identifying $(66,68)$ and $(93)$, one obtains:

$$
\left\{\begin{array}{l}
\nu_{-}^{\varepsilon}=a^{\varepsilon}\left[\frac{2}{\pi}\left(\log \frac{\omega \varepsilon}{2}+\gamma+\nu^{*}\right)+b^{*}\right]+u^{0}\left(A^{-}\right) \\
\nu_{+}^{\varepsilon}=(-1)^{\ell+1} a^{\varepsilon}\left[\frac{2}{\pi}\left(\log \frac{\omega \varepsilon}{2}+\gamma+\nu^{*}\right)+b^{*}\right]+u^{0}\left(A^{+}\right)
\end{array}\right.
$$

It follows from (92) and (94): $\quad a^{\varepsilon}=\frac{(-1)^{\ell} u^{0}\left(A^{+}\right)-u^{0}\left(A^{-}\right)}{\frac{4}{\pi}\left(\log \frac{\omega \varepsilon}{2}+\gamma+\nu^{*}\right)+2 b^{*}}$ 


\subsection{Towards a rigorous proof}

Since $a_{\varepsilon} \sim \frac{\pi}{4} \frac{(-1)^{\ell} u^{0}\left(A^{+}\right)-u^{0}\left(A^{-}\right)}{\log \varepsilon}$, we conjecture the estimate :

$$
\left\{\begin{array}{l}
\left\|u^{\varepsilon}-u^{0}\right\|_{L^{2}\left(\Omega_{R}\right)} \leqslant C\|f\|_{L^{2}(\Omega)} /|\log \varepsilon|, \\
\left\|u^{\varepsilon}-u^{0}\right\|_{L^{2}\left(O_{\varepsilon}\right)} \leqslant C\|f\|_{L^{2}(\Omega)} /\left(\varepsilon^{1 / 2}|\log \varepsilon|\right),
\end{array}\right.
$$

Moreover, due to the two logarithmic singularities at the point $A^{-}$and $A^{+}$, $u^{*}$ is not in $H_{l o c}^{1}(\Omega)$. Therefore we can not have the same inequality for the $H^{1}\left(\Omega_{R}\right)-$ norm as for the $L^{2}\left(\Omega_{R}\right)-$ norm. One can expect the (generically optimal) estimate:

$$
\left\|u^{\varepsilon}-u^{0}\right\|_{H^{1}\left(\Omega_{R}\right)} \leqslant C(\omega, L, R, F)\|f\|_{L^{2}(\Omega)} /|\sqrt{\log \varepsilon}| .
$$

All this results are coherent with those obtained numerically in section 3 . One can refer to figure 13, 14, 15, 16, 17, 18. The proof of (96) and (97) should follow the lines of the proof of theorem but the stability argument, that used the DtN map, has to be adequately modified. This paper is delayed to a forthcoming work.

\section{References}

[1] P. Joly, S. Tordeux, Asymptotic analysis of an approximate model for time harmonic waves in media with thin slots,.

[2] P. Harrington, D. Auckland, Electromagnetic transmission through narrow slots in thick conducting screens, IEEE Trans Antenna Propagation 28 (5) (1980) 616-622.

[3] G. Cohen, M. Duruflé, Mixed spectral elements for the helmholtz equation, in: G. C. Cohen, E. Heikkola, P. Joly, P. Neittaanmäki (Eds.), Proceedings of the Sixth International Conference on Mathematical and Numerical Aspects of Wave Propagation. Jyvaskyla, Finland., Springer-Verlag, 2003, pp. 743-748.

[4] D. Crighton, A. Dowling, J. F. Williams, M. Heckl, F. Leppington, Modern Methods in Analytical Acoustics, Lecture notes, Springer-Verlag, London, 1992.

[5] A. M. Il'in, Matching of asymptotic expansions of solutions of boundary value problems, Vol. 102 of Translations of Mathematical Monographs, American Mathematical Society, Providence, RI, 1992, translated from the Russian by V. Minachin [V. V. Minakhin]. 
[6] P. McIver, A. D. Rawlins, Two-dimensional wave-scattering problems involving parallel-walled ducts, Quart. J. Mech. Appl. Math. 46 (1) (1993) 89-116.

[7] S. Tordeux, Méthodes asymptotiques pour la propagation des ondes dans les milieux comportant des fentes, Ph.D. thesis, Université de Versailles (2004).

[8] M. Abramowitz, I. Stegun (Eds.), Handbook of mathematical functions with formulas, graphs, and mathematical tables, Dover Publications Inc., New York, 1992, reprint of the 1972 edition.

[9] S. Tordeux, Un problème de Laplace non standard en milieu non borné, C. R. Acad. Sci. Paris. 